\title{
Saturating field configurations for the massless Thirring-Wess model
}

\author{
H. O. Girotti* \\ Instituto de Física, Universidade Federal do Rio Grande do Sul, 90000 Porto Alegre, RS, Brasil
}

(Received 11 August 1978)

\begin{abstract}
The saturation of the massless Thirring-Wess model by induced field configurations is presented. The limiting procedure connecting the massless Thirring-Wess model with the Schwinger model is reexamined. Special emphasis is put on studying the dependence of saturating field configurations and topological charges on the vector-meson bare mass.
\end{abstract}

The saturation of Schwinger model ${ }^{1-3}$ Green's functions by induced instantons has been presented by Nielsen and Schroer in a recent series of papers. ${ }^{4-6}$ These induced instantons appear as solutions of an inhomogeneous field equation but only after performing the functional integration in the fermionic degrees of freedom. They are not the quasiclassical instantons of Belavin et $a l .,{ }^{7}$ but nevertheless they are responsible for the vaccum structure and the confinement properties exhibited by the Schwinger model.

On the other hand, Rothe and Swieca ${ }^{8,9}$ have pointed out that a better insight into the problem of confinement can be obtained by regarding the Schwinger model as the limit of a theory describing the dynamics of massless fermions coupled (coupling constant $e$ ) to massive vector mesons (bare mass $m_{0}$ ). This is the so-called massless Thirring-Wess (TW) model. ${ }^{10}$ In the limit $m_{0} \rightarrow 0$ the massless TW model goes into the Schwinger model.

Since the analysis of Rothe and Swieca ${ }^{8}$ is fully carried out at the operator level, we have found it interesting to reexamine the above-mentioned limit but now putting special emphasis on studying the $m_{0}$ dependence of saturating field configura- tions and associated topological charges (winding numbers).

The massless TW model is described by the Lagrangian density

$$
\begin{aligned}
\mathscr{L}= & -\frac{1}{4} F_{\mu \nu} F^{\mu \nu}+\frac{1}{2} m_{0}{ }^{2} B_{\mu} B^{\mu} \\
& +i \bar{\psi}(x) \gamma^{\mu}\left(\partial_{\mu}-i e B_{\mu}\right) \psi(x)-\frac{1}{2 a}\left(\partial_{\mu} B^{\mu}\right)^{2} .
\end{aligned}
$$

Here, $\psi(x)$ is a two-component massless fermion field, $B_{\mu}$ is a vector-meson field with bare mass $m_{0}$, and $F_{\mu \nu}$ is its corresponding field-strength tensor, i.e., $F_{\mu \nu}=\partial_{\mu} B_{\nu}-\partial_{\nu} B_{\mu}$. The term $-(1 / 2 a)\left(\partial_{\mu} B^{\mu}\right)^{2}$ has been added to maintain a well-defined theory in the limit $m_{0} \rightarrow 0$. Our conventions are

$$
\begin{aligned}
& x=\left(x^{0}, x^{1}\right), \\
& g^{00}=-g^{11}=\epsilon^{10}=\epsilon_{01}=1, \\
& \gamma^{0}=\left(\begin{array}{ll}
0 & 1 \\
1 & 0
\end{array}\right), \quad \gamma^{1}=\left(\begin{array}{cc}
0 & 1 \\
-1 & 0
\end{array}\right), \quad \gamma^{5}=\gamma^{0} \gamma^{1} .
\end{aligned}
$$

The massless TW model is solvable because the fermion propagator in an external $B_{\mu}$ field is exactly known. For the $2 n$-point fermion Green's function one finds ${ }^{4}$

$$
\begin{aligned}
&\left\langle\psi\left(x_{1}\right) \cdots \psi\left(x_{n}\right) \bar{\psi}\left(y_{1}\right) \cdots \bar{\psi}\left(y_{n}\right)\right\rangle= Z^{-1}\left\langle\psi^{(0)}\left(x_{1}\right) \cdots \psi^{(0)}\left(x_{n}\right) \bar{\psi}^{(0)}\left(y_{1}\right) \cdots \bar{\psi}^{(0)}\left(y_{n}\right)\right\rangle \\
& \times \int\left[D B_{\mu}\right] \exp \left[\frac{i}{2} \iint d^{2} z d^{2} z^{\prime} B^{\mu}(z) \Omega_{\mu \nu}^{(a)}\left(z, z^{\prime}\right) B^{\nu}\left(z^{\prime}\right)\right. \\
&\left.+i e \int d^{2} z B^{\mu}(z) \Sigma_{\mu}\left(z ; x_{1}, \ldots, x_{n} ; y_{1}, \ldots, y_{n}\right)\right] .
\end{aligned}
$$

Here, $\psi^{(0)}$ and $\bar{\psi}^{(0)}$ are free fermion fields, $\Sigma_{\mu}$ is a $B_{\mu}$-independent external-current-like factor containing $\gamma$ matrices and

$$
\Omega_{\mu \nu}^{(a)}\left(z, z^{\prime}\right)=\left[g_{\mu \nu} \square_{z}-\left(1-\frac{1}{a}\right) \partial_{z \mu} \partial_{z \nu}+g_{\mu \nu} m^{2}-\frac{e^{2}}{\pi} \partial_{z \mu} \square_{z}^{-1} \partial_{z \nu}\right] \delta^{(2)}\left(z-z^{\prime}\right),
$$

where to simplify the notation we have introduced the definitions

$$
m^{2}=m_{0}^{2}+e^{2} / \pi, \quad \partial_{z \mu}=\partial / \partial z^{\mu} .
$$

The functional integral in (2) is to be evaluated using vacuum-sector boundary conditions. The fermion renormalization constant $Z$ is chosen so as to have

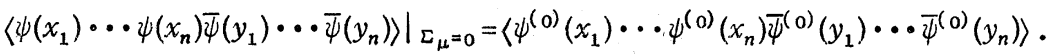


One should notice that the inverse of the symmetric kernel $\Omega_{\mu \nu}^{(a)}\left(z, z^{\prime}\right)$ yields the exact vector-meson propagator

$$
\Delta_{F \mu \nu}^{(a)}\left(z, z^{\prime}\right)=g_{\mu \nu} \Delta_{F}\left(z-z^{\prime} ; m^{2}\right)+\frac{1-a-\left(e^{2} / \pi\right) a \square_{z}^{-1}}{(1-a) m_{0}^{2}+e^{2} / \pi} \partial_{z \mu} \partial_{z \nu}\left[\Delta_{F}\left(z-z^{\prime} ; m^{2}\right)-\Delta_{F}\left(z-z^{\prime} ; a m_{0}^{2}\right)\right],
$$

where $\Delta_{F}\left(z-z^{\prime}, m^{2}\right)$ is the Green's function for a free massive scalar field, i.e.,

$$
\left(\square_{z}+m^{2}\right) \Delta_{F}\left(z-z^{\prime} ; m^{2}\right)=\delta^{(2)}\left(z-z^{\prime}\right) \text {. }
$$

We shall be interested first in the two-point function of the chiral density operators

$$
J_{\mp}(z)=N\left[\bar{\psi}(x) \frac{1 \pm \gamma_{5}}{2} \psi(x)\right] \text {. }
$$

By using Eq. (2) as a starting point, one arrives at

$$
\begin{aligned}
\left\langle J_{-}(x) J_{+}(y)\right\rangle=Z^{-1}\left\langle J_{-}^{(0)}(x) J_{+}^{(0)}(y)\right\rangle \int\left[D B_{\mu}\right] \exp & \left(\frac{i}{2} \iint d^{2} z d^{2} z^{\prime} B^{\mu}(z) \Omega_{\mu \nu}^{(a)}\left(z, z^{\prime}\right) B^{\nu}\left(z^{\prime}\right)\right. \\
+ & \left.i e \int d^{2} z B^{\mu}(z) H_{\mu}(z ; x, y)\right),
\end{aligned}
$$

where $J_{\mp}^{(0)}(x)$ are the chiral densities constructed from the free fermion field operators and

$$
H_{\mu}(z ; x, y)=2 \epsilon_{\mu \nu} \nu_{z}^{\nu}\left[D_{F}(z-x)-D_{F}(z-y)\right] .
$$

Here,

$$
D_{F}(z-x)=-\frac{i}{4 \pi} \ln \left(\left|\mu^{2}\right| z-\left.x\right|^{2}\right)+\text { const }
$$

is the free massless scalar field Green's function. As usual, infrared divergences are avoided by the introduction of an infrared regulator mass $\mu$. Since a finite renormalization will not alter our conclusions, we establish $\mu=m$.

There are two alternative procedures through which one can evaluate the functional integral in Eq. (6). This, of course, applies for any quadratic functional integral. The first procedure is straightforward and yields

$$
\left\langle J_{-}(x) J_{+}(y)\right\rangle=\left\langle J_{-}^{(0)}(x) J_{+}^{(0)}(y)\right\rangle \exp \left(-\frac{i}{2} e^{2} \iint d^{2} z d^{2} z^{\prime} H_{\mu}(z ; x, y) \Delta_{F}^{(a) \mu \nu}\left(z, z^{\prime}\right) H_{\nu}\left(z^{\prime} ; x, y\right)\right) .
$$

The second procedure consists of expanding the effective action functional

$$
S_{\text {eff }}\left[B^{\mu} \mid x, y\right]=\frac{1}{2} \iint d^{2} z d^{2} z^{\prime} B^{\mu}(z) \Omega_{\mu \nu}^{(a)}\left(z, z^{\prime}\right) B^{\nu}\left(z^{\prime}\right)+e \int d^{2} z B^{\mu}(z) H_{\mu}(z ; x, y)
$$

around the classical induced solution defined by

$$
\left.\frac{\delta S_{\mathrm{eff}}\left[B^{\mu} \mid x, y\right]}{\delta B^{\mu}(z)}\right|_{B^{\mu}=B_{\mathrm{cl}}^{\mu}}=0 \text {. }
$$

The zeroth order of the expansion is

$$
i S_{\text {eff }}\left[B_{\mathrm{cl}}^{\mu} \mid x, y\right] \text {. }
$$

The linear term in the quantum supplement $\eta^{\mu}\left(\eta^{\mu}=B^{\mu}-B_{\mathrm{cl}}^{\mu}\right)$ vanishes, while the quadratic term in $\eta^{\mu}$ is independent of $x$ and $y$ and, after performing the functional integration, it cancels against $Z^{-1}$. Therefore,

$$
\left\langle J_{-}(x) J_{+}(y)\right\rangle=\left\langle J_{-}^{(0)}(x) J_{+}^{(0)}(y)\right\rangle \exp \left\{i S_{\text {eff }}\left[B_{\mathrm{cl}}^{\mu} \mid x, y\right]\right\} .
$$

It is by looking at Eq. (12) that one states that $B_{\mathrm{cl}}^{\mu}$ saturates the two-point function $\left\langle J_{-}(x) J_{+}(y)\right\rangle$ in the massless TW model. From Eqs. (9) and (12) we have, as a consistency requirement, that

$$
S_{\text {eff }}\left[B_{\mathrm{cl}}^{\mu} \mid x, y\right]=-\frac{e^{2}}{2} \iint d^{2} z d^{2} z^{\prime} H_{\mu}(z ; x, y) \Delta_{F}^{(a) \mu \nu}\left(z, z^{\prime}\right) H_{\nu}\left(z^{\prime} ; x, y\right) \text {. }
$$

Our next step is to find $B_{\mathrm{cl}}^{\mu}$. The equation of motion for $B_{\mathrm{cl}}^{\mu}$ is obtained from Eqs. (10) and (11) and it reads 


$$
\int d^{2} z^{\prime} \Omega_{\mu \nu}^{(a)}\left(z, z^{\prime}\right) B_{\mathrm{cl}}^{\mu}\left(z^{\prime} ; x, y\right)=-e H_{\mu}(z ; x, y)
$$

whose solution is found to be

$$
B_{\mathrm{cl}}^{\mu}(z ; x, y)=B_{+}^{\mu}(z ; y)+B_{-}^{\mu}(z ; x),
$$

where

$$
\begin{aligned}
& B_{+}^{\mu}(z ; y)=\frac{2 e}{m^{2}} \epsilon^{\mu \nu} \partial_{z \nu}\left[D_{F}(z-y)-\Delta_{F}\left(z-y ; m^{2}\right)\right], \\
& B_{-}^{\mu}(z ; x)=-\frac{2 e}{m^{2}} \epsilon^{\mu \nu} \partial_{z \nu}\left[D_{F}(z-x)-\Delta_{F}\left(z-x ; m^{2}\right)\right] .
\end{aligned}
$$

We remark that $B_{\mathrm{cl}}^{\mu}$ is independent of the gauge parameter $a$ and, therefore, so is $S_{\mathrm{eff}}\left[B_{\mathrm{cl}}^{\mu} \mid x, y\right]$. One can check that the same is true for the right-hand side of (13). In fact

$$
\begin{aligned}
S_{\mathrm{eff}}\left[B_{\mathrm{cl}}^{\mu} \mid x, y\right] & =-\frac{e^{2}}{2} \iint d^{2} z d^{2} z^{\prime} H_{\mu}(z ; x, y) \Delta_{F}^{(a) \mu \nu}\left(z, z^{\prime}\right) H_{\nu}\left(z^{\prime} ; x, y\right) \\
& =\frac{4 e^{2}}{m^{2}}\left[D_{F}(x-y)-\Delta_{F}\left(x-y ; m^{2}\right)\right]
\end{aligned}
$$

as required.

We now look into the topological content of the field configurations $B_{+}$and $B_{-}$. The corresponding winding numbers (topological charges), $n_{+}$and $n_{-}$, respectively, are given by the following expressions $^{11}$ :

$$
n_{ \pm}= \pm \frac{e^{2} / \pi}{m^{2}}= \pm \frac{e^{2} / \pi}{m_{0}^{2}+e^{2} / \pi} .
$$

Thus one arrives at the conclusion that in the massless TW model the two-point function $\left\langle J_{-}(x) J_{+}(y)\right\rangle$ is saturated by a pair of field configurations each with a nonvanishing topological charge. Note that the winding numbers $n_{ \pm}$depend continuously upon $m_{0}$ and that they tend to zero in the limit of extremely massive vector mesons $\left(m_{0} \rightarrow \infty\right)$.

Our immediate task is to investigate the possible implications of the topology, namely, the vacuum structure. The behavior of $\left\langle J_{-}(x) J_{+}(y)\right\rangle$ for large spacelike separations can be found from Eqs. (12) and (17). One easily arrives at

$\left\langle J_{-}(x) J_{+}(y)\right\rangle$

$$
\underset{|x-y| \rightarrow \infty}{\sim} \text { const } \times\left(|x-y|^{2}\right)^{-m_{0}^{2} /\left(m_{0}^{2}+e^{2 / x}\right)} .
$$

Hence, for $m_{0}>0$,

$$
\lim _{|x=y| \rightarrow \infty}\left\langle J_{-}(x) J_{+}(y)\right\rangle=0 \text {. }
$$

As one can see the two-point function $\left\langle J_{-}(x) J_{+}(y)\right\rangle$ verifies the cluster decomposition theorem and, therefore, no chiral vacuum degeneracy arises.

Then, in the massless TW model one has a nontrivial topology coexisting with a unique vacuum. Somehow this is the theoretical counterpart of the situation in the Schwinger model.

In the limit $m_{0} \rightarrow 0(m \rightarrow e / \sqrt{\pi})$ a radical change arises. In this case (Schwinger model) the behavior of $\left\langle J_{-}(x) J_{+}(y)\right\rangle$ for large spacelike separations is no longer given by Eq. (20). In fact, if one replaces $m_{0}=0$ in Eq. (19) one obtains

$$
\lim _{|x=y| \rightarrow \infty}\left\langle J_{-}(x) J_{+}(y)\right\rangle=\text { const } \neq 0 .
$$

Then, in the limit $m_{0} \rightarrow 0$ the cluster property breaks down and, as a consequence, the vacuum degenerates. ${ }^{4}$ Also note [see Eq. (18)] that for $m_{0}=0$ the winding numbers $n_{ \pm}$, associated with the saturating field configurations $B_{+}^{\mu}$ and $B_{-}^{\mu}$, respectively, reach their maximum absolute values, i.e.,

$$
\left.n_{ \pm}\right|_{m_{0}=0}= \pm 1 \text {. }
$$

Needles to say, the analytic continuation of Eqs. (16) into Euclidean space yield, after setting $m=e / \sqrt{\pi}$, the instanton-antiinstanton pair already found by Nielsen and Schroer. ${ }^{5}$

Our second object of interest is the two-point fermion Green's function $\langle\psi(x) \bar{\psi}(y)\rangle$, which should reveal to us the confinement properties of the theory. For the massless TW model one finds, for $a=0$, that

$$
\left\langle\psi_{1}(x) \bar{\psi}_{1}(y)\right\rangle=\left\langle\psi_{1}^{(0)}(x) \bar{\psi}_{1}^{(0)}(y)\right\rangle \exp \left\{\frac{i e^{2}}{m^{2}}\left[D_{F}(x-y)-\Delta_{F}\left(x-y ; m^{2}\right)\right]\right\},
$$

where $\psi^{(0)}(x)$ and $\bar{\psi}^{(0)}(y)$ are free fermion fields. The subscripts in (23) refer to spin components. 
Once again one can write

$$
\left\langle\psi_{1}(x) \bar{\psi}_{1}(y)\right\rangle=\left\langle\psi_{1}^{(0)}(x) \bar{\psi}_{1}^{(0)}(y)\right\rangle \exp \left(i \tilde{S}_{\mathrm{eff}}\left[\tilde{B}_{\mathrm{cl}}^{\mu} \mid x, y\right]\right),
$$

where the new effective action $\widetilde{S}_{\text {eff }}$ is given by

$$
\begin{aligned}
\tilde{S}_{\mathrm{eff}}\left[B^{\mu} \mid x, y\right]= & \frac{1}{2} \iint d^{2} z d^{2} z^{\prime} B^{\mu}(z) \Omega_{\mu \nu}^{(a)}\left(z, z^{\prime}\right) B^{\nu}\left(z^{\prime}\right) \\
& -e \int d^{2} z B^{\mu}(z)\left(\partial_{z \mu}-\epsilon_{\mu \nu} \partial_{z}^{\nu}\right)\left[D_{F}(z-x)-D_{F}(z-y)\right] .
\end{aligned}
$$

This time the saturating field configuration, $\vec{B}_{\mathrm{cl}}^{\mu}$, depends on $a$ and is found to read

$$
\tilde{B}_{\mathrm{cl}}^{\mu}(z ; x, y)=\tilde{B}_{+}^{\mu}(z ; y)+\tilde{B}_{-}^{\mu}(z ; x),
$$

where

$$
\begin{aligned}
& \tilde{B}_{+}^{\mu}(z ; y)=\frac{e}{m^{2}} \epsilon^{\mu \nu} \partial_{z \nu}\left[D_{F}(z-y)-\Delta_{F}\left(z-y ; m^{2}\right)\right]-\frac{e}{m_{0}^{2}} \partial_{z}^{\mu}\left[D_{F}(z-y)-\Delta_{F}\left(z-y ; a m_{0}^{2}\right)\right], \\
& \tilde{B}_{-}^{\mu}(z ; x)=-\frac{e}{m^{2}} \epsilon^{\mu \nu} \partial_{z \nu}\left[D_{F}(z-x)-\Delta_{F}\left(z-x ; m^{2}\right)\right]+\frac{e}{m^{2}} \partial_{z}^{\mu}\left[D_{F}(z-x)-\Delta_{F}\left(z-x ; a m_{0}^{2}\right)\right] .
\end{aligned}
$$

The effective action $\vec{S}_{\text {eff }}$ evaluated at the solution $\tilde{B}_{\mathrm{cl}}^{\mu}$ also depends on the gauge parameter $a$. One easily checks that, for $a=0$,

$$
\tilde{S}_{\mathrm{eff}}\left[\tilde{\boldsymbol{B}}_{\mathrm{cl}}^{\mu} \mid x, y\right]=\frac{e^{2}}{m^{2}}\left[D_{F}(x-y)-\Delta_{F}\left(x-y ; m^{2}\right)\right],
$$

as required [see Eqs. (23) and (24)].

The $a$-dependent part of the field configurations $\tilde{B}_{+}^{\mu}$ and $\tilde{B}_{-}^{\mu}$ is fully contained in the longitudinal terms. Hence, the winding numbers $\tilde{n}_{+}$and $\tilde{n}_{-}$, corresponding to the field configurations $\tilde{B}_{+}^{\mu}$ and $\tilde{B}_{-}^{\mu}$, respectively, are independent of $a$. One finds that ${ }^{11}$

$$
\tilde{n}_{ \pm}= \pm \frac{1}{2} \frac{e^{2} / \pi}{m^{2}}= \pm \frac{1}{2} \frac{e^{2} / \pi}{m_{0}^{2}+e^{2} / \pi}
$$

Then, in the massless TW model, where fermion confinement does not arise, 8.9 the two-point fermion Green's function is saturated by a pair of field configurations each with nonvanishing topological charge.

In the limit $m_{0} \rightarrow 0$, where fermion confinement takes place (Schwinger model), ${ }^{2,3,8,9}$ the winding numbers $\tilde{n}_{ \pm}$acquire their maximum absolute values

$$
\left.\tilde{n}_{ \pm}\right|_{m_{0=0}= \pm \frac{i}{2}}
$$

After analytic continuation into Euclidean space, the transversal parts of $\tilde{B}_{ \pm}^{\mu}$ go, for $m_{0}=0$, into the transversal parts of the $c$ instanton and $c$ antiinstanton, respectively (see Ref. 6). As far as the longitudinal parts are concerned our results differ from the corresponding ones in Ref. 6 . These differences can be traced back to the absence of the term

$$
\exp \left(-i e \int_{y}^{x} B^{\mu}(s) d s_{\mu}\right)
$$

in our definition of the two-point function $\left\langle\psi_{1}(x) \bar{\psi}_{1}(y)\right\rangle$. That the introduction of this term can be avoided has been pointed out by Rothe and Swieca. ${ }^{12}$

We have come to an end in our particular analysis of the limiting procedure connecting the massless TW model with the Schwinger model. The situation can be summarized as follows. The topological ingredient is always present except, of course, for $m_{0} \rightarrow \infty$. A nonvanishing mechanical mass for the vector mesons $\left(m_{0}>0\right)$ implies explicit breaking of second-kind gauge invariance and a limitation in the absolute values of the winding numbers associated with the saturating field configurations. In this case $\gamma_{5}$ invariance is a good symmetry and fermions remain unconfined. For $m_{0}=0$ one has spontaneous (and non-Goldstone) symmetry breaking of gauge and $\gamma_{5}$ invariance. The winding numbers reach their maximum absclute values and fermion confinement takes place.

Only in the limit $m_{0} \rightarrow 0$ does the topological content of the theory become strong enough to give rise to a nontrivial vacuum structure and to fermion confinement. It is interesting to note that in the limit $m_{0} \rightarrow 0$ the magnitude of the topological charges becomes exactly tuned with the class index of the gauge transformations connecting two successive vacuums in the Schwinger model. 12,13

I am indebted to Professor Th. A. J. Maris and to Professor J. A. Swieca for discussions. This work was supported in part by Financiadora de Estudos e Projetos (Brasil). 
*On leave of absence from Consejo Nacional de Investigaciones Cientificas y Tecnicas de Argentina.

${ }^{1} \mathrm{~J}$. Schwinger, Phys. Rev. 128, 2425 (1962); in Theoretical Physics, Trieste Lectures, Vienna 1962 (T. A.E. A. , Vienna, 1963), p. 89

${ }^{2} J$. H. Lowenstein and J. A. Swieca, Ann. Phys. (N. Y.) 68, 172 (1971).

${ }^{3}$. Casher, J. Kogut, and L. Susskind, Phys. Rev. D 10, 732 (1974).

${ }^{4}$ N. K. Nielsen and B. Schroer, Nucl. Phys. B120, 62 (1977).

${ }^{5}$ N. K. Nielsen and B. Schroer, Phys. Lett. $66 \mathrm{~B}, 373$ (1977).

${ }^{6}$ N. K. Nielsen and B. Schroer, Phys. Lett. $66 \mathrm{~B}, 475$ (1977).
${ }^{7}$ A. A. Belavin, A. M. Polyakov, A. S. Schwartz, and Yu. S. Tyupkin, Phys. Lett. 59B, 85 (1975).

${ }^{8}$ K. D. Rothe and J. A. Swieca, Phys. Rev. D 15, 1675 (1977).

${ }^{9} \mathrm{~J}$. A. Swieca, Fortsch. Phys. 25, 303 (1977).

${ }^{10} \mathrm{~W}$. E. Thirring and J. E. Wess, Ann. Phys. (N.Y.) 27, 331 (1964).

${ }^{11}$ C. G. Callan, R. Dashen, and D. Gross, Phys. Lett. 63B, 334 (1976).

${ }^{12}$ K. D. Rothe and J. A. Swieca, PUC Rio de Janeiro report 1978 (unpublished); and J. A. Swieca, private communication.

${ }^{13}$ K. D. Rothe and J. A. Swieca, Phys. Rev. D $\underline{15}, 541$. (1977). 